\title{
Evaluation of the prognostic value of multimodal intraoperative monitoring in posterior fossa surgery patients with cerebellopontine angle tumors
}

Mohsen Dalvandi (1), Ali Nazemi Rafie (1), Alireza Kamali (2), Alireza Jamshidifard (3)

(1) Department of Neurosurgery, Arak University of Medical Sciences, Arak; (2) Department of Anesthesiology, Arak University of Medical Sciences, Arak; (3) Department of Neurology, Arak University of Medical Sciences, Arak Iran.

This article is distributed under the terms of the Creative Commons Attribution Noncommercial License (CC BY-NC 4.0) which permits any noncommercial use, distribution, and reproduction in any medium, provided the original author(s) and source are credited.

\begin{abstract}
The aim of this study was to compare the outcomes of four intraoperative monitoring approaches in order to compare their prognostic value in surgical complications of the facial nerve. This quasi-experimental study was conducted on 25 patients with Cerebellopontine Angle (CPA) tumors, who were hospitalized in the Valiasr Hospital, affiliated to the Arak University of Medical Sciences, Arak, Iran. The degree of the facial nerve paralysis was assessed based on the House-Brackmann (HB) facial nerve grading system. The Orbicularis oris and Oculi muscles were evaluated by electromyography (EMG) before and after the surgery. Monitoring the facial nerve was performed by the EMG, Facial Nerve Antidromic Potentials (FNAPs), Transcranial Electrical Stimulation (TES), and Brainstem Auditory Evoked Potentials (BAEPs) methods. The mean HB score was $0.36 .6 \pm 5.83$, indicating that the function of the facial muscle was abnormal in all the patients pre-surgery. The comparison of the four monitoring approaches six months after surgery showed that the predictive value of facial nerve TES was higher than the other approaches $(\mathrm{P}<0.005)$. Furthemroe, $4 \%$ of the patients showed normal function in the facial muscle after the surgery. In addition, $8 \%, 56 \%$, and $32 \%$ of the patients had mild abnormalities, moderate to severe paralysis in the facial muscles, and severe facial nerve dysfunction, respectively. In summary, $36 \%$ of the patients showed a mild abnormalities in the follow-up period, and full recovery was observed in $28 \%$ of the cases. In conclusion, the transcranial motor evoked potential (TCMEP) is a useful method for monitoring the facial nerve during the surgery along with the continuous EMG recordings.
\end{abstract}

Key Words: Multimodal IOM, CPA Tumor Resection, Facial Nerve, Acoustic nerve, Predictive Value

Eur J Transl Myol 28 (1): 124-131, 2018

$\mathbf{O}_{\text {ne of the main problems of the neurological surgery is }}$ the damage to the peripheral or central nerves. Cerebellopontine angle (CPA) tumors are the most common neoplasm in the posterior fossa, which can lead to unilateral hearing loss, speech impediments, disequilibrium, and other loss of motor control. ${ }^{1}$ In some cases such as those with meningioma, the patient's life is seriously at risk; therefore, the therapeutic measures should be performed as soon as possible for these patients. $^{2,3}$ There are some factors playing an important role in the treatment process of this tumor. These factors include age, tumor size, time of tumor diagnosis, cranial nerve condition before surgery, surgical route, the adhesion of the facial nerve sheath, and previous underlying diseases 4. Damage to the cranial nerve during tumor removal is inevitable in some cases, especially in the tumors bigger than $4 \mathrm{~cm}$. Therefore, the facial nerve damage may be widespread, leading to practical problems and changing the emotional and social factors in the patient's future life.

The use of the intraoperative neurophysiological monitoring (IOM) is a common approach to monitor the functional integrity of certain neural structures during the neurological surgery. This approach aims to pervent the damage caused by the manipulation of the nervous tissue, ${ }^{5,6}$ however, it is not very common in Iran compared to other countires. 
Monitoring the facial nerve during the removal of such tumors as vestibular schwannoma and other posterior fossa tumors can help not only reduce the post-surgery facial nerve damage, but also improve the patient's quality of life and decrease the further medical costs. Therefore, it is necessary to perform some studies assessing the exact criteria for monitoring and determining the respective applicable approaches. In this regard, no accurate method has been proposed for estimating the prognostic value of these approaches in facial nerve function after the CPA surgery. EMG is a well-known technique for monitoring the facial nerve during the CPA operation. This method is used for the registration of facial muscle responses to the electrical and mechanical stimulations. ${ }^{7}$ However, due to the excessive sensitivity of EMG technique in the administration of neuromuscular blockers, the application of additional methods is required. Antidromic facial nerve potentials (FNAPs), transcranial motor evoked potentials (TCMEPs), and brainstem auditory evoked potentials (BAEPs) are other methods for continuous monitoring of the facial nerve. ${ }^{8}$. All these methods are effective in the prevention of the damage during the surgery and the prediction of the patient's condition post-surgery. Despite the growing trend of IOM in the cervical spine surgery, there is limited data on the sensitivity, specificity, and predictive values of these techniques in detecting new neurological deficits. Although IOM has a long history, its application has only recently become widespread. ${ }^{9}$ Accordingly, the commercial IOM machine and its educational programs were recently developed in Iran. Although IOM has no impact on the pathology of the patients, it can affect the process of maintaining the anatomical integrity during the surgery. With this background in mind, the present study aimed to compare the functions of the four intraoperative monitoring approaches in order to compare their prognostic values in surgical complications of the facial nerve.

\section{Materials and Methods}

\section{Ethical Approval}

The present study was performed based the Codes of Medical Ethics released by the Ministry of Health and Medical Education, Iran and Declaration of Helsinki. In this study, all approaches were performed based on the international standard protocol. The stages and techniques of this study were clearly explained to the patients, and their informed consent was obtained. Moreover, they were assured about the confidentiality of their information and the possibility to leave the study at any stage of the project. In addition, no money was obtained from the patients until the sixth month of operation.

\section{Participants}

This quasi-experimental study was conducted on 32 patients with CPA tumor, who were hospitalized in the
Valiasr hospital affiliated to the Arak University of Medical Sciences, Arak, Iran. Out of the 32 patients selected by the convenience sampling method, 25 cases were candidates for surgery. The exclusion criteria included suffering from congenital deafness, facial paralysis, diabetes mellitus, or addiction, and those undergoing radio or re-surgery six months after surgery.

\section{Study design}

The patients were selected based on the inclusion criteria to minimize the sampling error or bias risk. In the first step, the degree of the facial nerve paralysis was assessed based on the House-Brackmann (HB) facial nerve grading system. Additionally, the Orbicularis oris and Oculi muscles were evaluated by the EMG prior to the surgery. To determine the type and size of tumor, certain para-clinic tests, such as computed tomography scan and magnetic resonance imaging with or without contrast, were performed by neurologist colleagues. Subsequently, the patients were referred for the surgery. In accordance with the monitoring methods, the anesthetic technique was performed using the Los Angeles Anesthesia Friendliness Scale protocol (modified in 5/17/2004). The resection surgery was performed through the retrosigmoid and middle fossa approaches. The facial VII and acoustic VIII nerves were assessed before, during, and six months after the surgery. Due to the lack of data normality, the obtained data of each patient was paired with his/her data in the four stages. To minimize the bias risk of other confounding variables, the temperature of operating room and that of the rooms the patients were kept in before and after operation was fixed at $25^{\circ} \mathrm{C}$. Furthermore, all the patients were operated by the same surgeon. During surgery, the antidromic sensory nerve action potentials of the facial nerve in the CPA were repeatedly recorded by the FNAPs method. The electrical stimulation was performed by corkscrew on the $\mathrm{Cz}, \mathrm{C} 3$, and $\mathrm{C} 4$ regions through the transcranial electrical stimulation (TES) approach. The motor evoked potentials (MEP) of the Orbicularis Oris and Oculi muscles were recorded by the EMG. About four to six transcranial stimulations were performed (typically at the intervals of 2-4 ms) with an intensity of 300-500 mA in the pre-specified regions of the skull by the TES approach. The location of the electrodes during the intraoperative electromyographic monitoring of cranial nerves is shown in Table 1 . The extended and freerunning EMG of the orbicularis oris and oculi muscles were also recorded during the surgery. The EMG activity in response to direct electrical stimulation of nerve and spontaneous activities were recorded using a monitoring device (Viasys/Nicolet, USA) containing 52 canals. The other invwstigations were performed pre- and postsurgery by the EMG and BAEP evaluation through electrodiagnostic 4-channel system. These devices were placed in the Laboratory of Clinical Neurophysiology at Medical School of Mashhad University of Medical 
Table 1. Location of the electrodes in intraoperative electromyographic monitoring of cranial

\begin{tabular}{ll}
\hline Cranial nerve & Muscle \\
\hline V & Masseter \\
VII & Frontalis, Zygomaticus, \\
& Buccalis, Mentalis \\
IX & Soft palate \\
X & Vocal cord \\
XI & Trapezius \\
\hline
\end{tabular}

Sciences, Mashhad, Iran, and neurosurgical operating rooms of Valiasr Hospital. The stimulation parameters and recording process were controlled by the computers connected to the device. Time and amplitude parameters were listed based on $\mathrm{mSe}$ and $\mathrm{mV}$, respectively, recorded to two decimal places on the computer screen. Our devices were equipped with amplifier system and digital converter; therefore, they were automatically calibrated in each registration stage. Prior to surgery, pure tone and absolute threshold of hearing (ATH) were measured. Subsequently, the speech discrimination score (SDS) was obtained, which was repeated three weeks and six months after the surgery. The SDS was standardized by Moslehi et al. in $2001 .^{10}$. The BAEPs of both ears were recorded by stimulating the electrodes fixed in the patients' ears before, during, and after the surgery. The auditory status was monitored by the IOM device and the use of installed speakers of ear canal under surgery providing 31 stimulations per second, which was simultaneously recorded by the electrodes placed on the scalp from both ear canals. The electrodes were placed in both ears and vertex $(\mathrm{Cz})$, and the filtration was adjusted within 150 3,000 HZ. The mean of each recorded signal of BAEP was 1,000-4,000 stimulations, which was repeated

Table 2. Change of the facial nerve before and after surgery based on House-Brackmann facial nerve grading scale

\begin{tabular}{llll}
\hline Cases & Pre-surgery & Post-surgery & \\
\cline { 2 - 3 } & & After 3 weeks & After 6 weeks \\
& & & \\
1 & 0.875 & 1 & 1 \\
2 & 0.625 & 0.75 & 0.875 \\
3 & 0.75 & 0.5 & 1 \\
4 & 0.625 & 0.125 & 1 \\
5 & 0.375 & 0.75 & 0.625 \\
6 & 0.75 & 0.125 & 1 \\
7 & 0.125 & 0.25 & 0.875 \\
8 & 0.125 & 0 & 0.875 \\
9 & 0.25 & 0 & 0.75 \\
10 & 0.125 & 0.25 & 0.875 \\
11 & 0.25 & 0.25 & 0.875 \\
12 & 0.5 & 0.875 & 1 \\
13 & 0.125 & 0 & 0.875 \\
14 & 0.25 & 0 & 0.75 \\
15 & 0.25 & 0.125 & 0.875 \\
16 & 0.125 & 0.25 & 0.625 \\
17 & 0.5 & 0.25 & 0.875 \\
18 & 0.375 & 0.875 & 1 \\
19 & 0.875 & 0.5 & 1 \\
20 & 0.75 & 0.5 & 1 \\
21 & 0.25 & 0 & 0.75 \\
22 & 0.25 & 0 & 0.625 \\
23 & 0.375 & 0.25 & 0.875 \\
24 & 0.25 & 0 & 0.75 \\
25 & 0.0 & 0 & 0.75 \\
\hline
\end{tabular}


almost every $2 \mathrm{~min}$. The presence of signal and wave $\mathrm{V}$ latency were reported. The changes of $\mathrm{V}$ waveform and the lack or presence of wave V latency (more than $1 \mathrm{~m}$ ) were reported to the surgeon. As a result, the surgeon was recommended to change the position of the retractor on the cerebellum and reduce its stretch as long as the change in signal be compensated. This lasted for a few seconds, and the signal appeared again. In the BAEP technique, more than a millisecond delay for the wave $\mathrm{V}$ latency was considered significant. Moreover, the audiometry, pure-tone average, and SDS were evaluated and recorded for each patient before and after the surgery by an audiologist. Based on the HB scale, the degree of the facial nerve paralysis of the Orbicularis and Oculi muscles was evaluated through the EMG in the third week and sixth month of the surgery. All data were registered in the medical records of the patients, and then analyzed in the EXCEL and SPSS software using the data regression tests.

\section{Results}

About $76 \%$ of the patients were female. The mean age of the participants was $50.05 \pm 10.81$ years. Out of the 25 subjects, 23 patients were diagnosed with vestibular schwannoma, 1 with neurofibromatosis, and 1 with recurrent tumor. Furthermore, meningioma was observed in two males. The mean tumor size was $36.64 \pm 5.83 \mathrm{~mm}$, ranging within $24-45 \mathrm{~mm}$. About $76 \%$ and $24 \%$ of the tumors were removed through the retro-sigmoid and middle cranial fossa approaches, respectively. The change of the facial nerve paralysis degree before and after the surgery based on the HB is shown in Table 2. The mean HB score was 0.36.6 \pm 5.83 , therefore, the function of the facial muscle was abnormal in all the patients prior to the surgery. Before the surgery, the mean values of ATH and SDS were $43.58 \pm 15.97 \mathrm{~dB}$ and $38.08 \pm 10.43$ in the affected side, respectively. Six months after the surgery, the mean ATH was $10.49 \pm 31.76 \mathrm{~dB}$, which was increased in the affected side of eight patients and decreased in seventeen patients. Furthermore, the mean of SDS was 59.92 \pm 13.96 after six months, showing an increase in the affected side of the patients. The comparison of ATH scores showed that there was a significant difference in the hearing quality before and after the operation $(\mathrm{P}<0.005)$. Likewise, the SDS was significantly different before and after the surgery $(\mathrm{P}<0.005)$. However, the ATH and SDS scores showed no correlation with the HB score before and after the operation $(\mathrm{P}>0.005)$. The comparison of the facial nerve paralysis degree of the patients six months after the surgery demonstrated no significant relationship between the EMG findings and spikes recorded in the free-running EMG. Furthermore, there was no significant relationship between the facial status and latency changes of BAEP wave and the presence or lack of wave V. The changes of audiology test results in both ears before and after the surgery are shown in Table 3. There was a weak correlation between the HB score and amplitude response of FNAP during the surgery before removing the tumor $(r=0.67, P=0.005)$. The evaluation of the reflexes and evoked responses of Orbicularis Oris and Oculi after the operation and six months after tumor removal revealed a direct weak correlation between the $\mathrm{HB}$ score and amplitude response of FNAP $(\mathrm{r}=0.59, \mathrm{P}=0.005)$. Additionally, a positive correlation was observed before removing the tumor and six months after the operation between the HB score and TES amplitude response of facial muscles $(r=0.78, P=0.005)$. Similarly, a high direct correlation was observed between the HB score and TES amplitude response of facial muscles in the affected side and normal side of each patient after tumor removal ( $\mathrm{r}=0.807, \mathrm{P}=0.005)$.

The positive prognostic values of the four monitoring approaches were measured using the following formula:

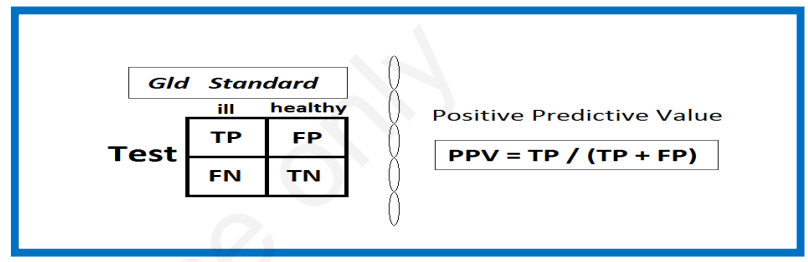

The predictive values of anti-dromic facial nerve stimulation, facial nerve TES, free-running EMG, and BAER were $36.3,0.87,0.43$, and 0.6 , respectively. The comparison of these four approaches six months after the surgery showed that the predictive value of the facial nerve TES was higher than those of the other approaches. In the patients with the HB score of one, a low incidence of facial nerve involvement was reported postsurgery.However, in some cases, the recovery of facial nerve function was observed immediately after the surgery. In addition, all the patients were found to have such recovery six months after the operation, rendering the mean $\mathrm{HB}$ of $0.86 \pm 0.12$ post-surgery. Following the surgery and getting discharged from the hospital, $4 \%$ of the patients were observed to have normal function in the facial muscle. In addition, $8 \%, 56 \%$, and $32 \%$ of the patients had mild abnormalities, moderate to severe paralysis in the facial muscles, and severe facial nerve dysfunction, respectively. In summary, $36 \%$ of the patients showed mild abnormalities in the follow-up, and full recovery was observed in $28 \%$ of the cases. Furthermore, two patients one of whom suffering from Ependymoma and the other one having a history of Cerebral Vascular Accident passed away during the follow-up due to bleeding in the brain stem and spontaneous bleeding unrelated to the surgery on the opposite side (in the supratentorial), respectively.

\section{Discussion}

As the findings of our study indicate, the transcranial stimulation of the facial nerve is a useful technique to monitor the facial nerve performance during CPA operation. Other similar studies have also confirmed this finding. ${ }^{411-13}$ Our data showed that the hearing quality 
Prognostic Value of Multimodal Intraoperative Monitoring

Eur J Transl Myol 28 (1): 124-131, 2018

Table 3. Changes of audiology test results in both ears before and after the surgery

\begin{tabular}{|c|c|c|c|c|c|c|c|c|}
\hline \multirow[t]{4}{*}{ Cases } & \multicolumn{8}{|c|}{ Six months post-surgery } \\
\hline & \multicolumn{2}{|c|}{ PTA audiometry (dB) } & \multicolumn{2}{|r|}{ SDS (\%) } & \multicolumn{2}{|c|}{ PTA audiometry (dB) } & \multirow{3}{*}{$\begin{array}{r}\text { Normal } \\
\text { side }\end{array}$} & \multirow{3}{*}{$\begin{array}{r}\text { SDS (\%) } \\
\text { Affected } \\
\text { side }\end{array}$} \\
\hline & Normal & Affected & Normal & Affected & Normal & Affected & & \\
\hline & side & side & side & side & side & side & & \\
\hline 1 & 8.16 & 26.28 & 64 & 52 & 8 & 43 & 64 & 56 \\
\hline 2 & 12.64 & 22.45 & 72 & 64 & 12.21 & 54 & 72 & 72 \\
\hline 3 & 10.58 & 27.34 & 66 & 42 & 10.32 & 40 & 66 & 54 \\
\hline 4 & 5.9 & 24.64 & 60 & 46 & 6 & 37 & 60 & 54 \\
\hline 5 & 8.6 & 38.46 & 52 & 32 & 8 & 62 & 52 & 44 \\
\hline 6 & 11.5 & 38.82 & 68 & 48 & 10 & 35 & 68 & 56 \\
\hline 7 & 5.7 & 36.80 & 76 & 44 & 5.3 & 30 & 76 & 56 \\
\hline 8 & 9.7 & 41.34 & 68 & 32 & 9.5 & 22 & 68 & 48 \\
\hline 9 & 9.4 & 46.62 & 42 & 30 & 9.7 & 26 & 42 & 46 \\
\hline 10 & 12.3 & 40.86 & 54 & 38 & 12.5 & 30 & 54 & 50 \\
\hline 11 & 7.4 & 48.22 & 44 & 28 & 7 & 20 & 44 & 40 \\
\hline 12 & 6.6 & 58.36 & 54 & 36 & 6.2 & 32 & 54 & 52 \\
\hline 13 & 7.9 & 47.93 & 42 & 22 & 8 & 18 & 42 & 44 \\
\hline 14 & 14.3 & 52.65 & 56 & 30 & 14.5 & 30 & 56 & 40 \\
\hline 15 & 5.9 & 70.62 & 60 & 34 & 6 & 34 & 60 & 54 \\
\hline 16 & 10.4 & 48.38 & 64 & 36 & 10 & 32 & 64 & 48 \\
\hline 17 & 11.7 & 39.18 & 70 & 40 & 12 & 36 & 70 & 56 \\
\hline 18 & 9.7 & 30.41 & 78 & 48 & 9.5 & 36 & 78 & 64 \\
\hline 19 & 8.5 & 20.36 & 86 & 56 & 8.5 & 35 & 86 & 64 \\
\hline 20 & 6.8 & 18.38 & 88 & 48 & 7 & 26 & 88 & 80 \\
\hline 21 & 7.8 & 68.92 & 46 & 32 & 8 & 24 & 46 & 50 \\
\hline 22 & 11.6 & 66.38 & 36 & 28 & 11 & 30 & 36 & 36 \\
\hline 23 & 13.4 & 70.36 & 54 & 32 & 13 & 20 & 54 & 40 \\
\hline 24 & 11.9 & 44.86 & 56 & 28 & 12 & 24 & 56 & 52 \\
\hline 25 & 8.5 & 60.88 & 42 & 26 & 8.2 & 18 & 42 & 40 \\
\hline
\end{tabular}

PTA: pure-tone average, SDS: speech discrimination score

and speech discrimination were improved six months after the operation. Furthemore, a high direct correlation was observed between the HB score and TES amplitude response of facial muscles in the affected side and normal side of the patients after tumor removal. In our study, the TCMEP was performed to measure the optimal stimulation parameters of the facial nerve by using multipulse stimulation of 3-5-pulse trains, duration of $0.2-0.5 \mathrm{~ms}$, and interstimulus interval of $4 \mathrm{~ms}$ as performed in the pervious studies..$^{411-13}$. The comparison of the affected and normal sides of facial nerve gives an optimal estimation from the nerve clinical practice. In our study, the highest electromyographic responses between the two sides of facial nerve were observed when comparing the TES responses of facial muscles. Therefore, the post-surgery stimulation of motor cortex of facial nerve results in improved state of descending motor pathways. The TCMEP findings had the highest correlation with the six-month follow-up results than the other approaches. Regarding this, the TCMEP can be 
introduced as a successful intraoperative neural monitoring in case it is applied by an experienced neuroanesthesiology team. Moreover, the TCMEP data are useful in distinguishing the subjects with initial paresis/paralysis as well as the recovery of their facial function in the long-term. Cosetti et al. evaluated the efficiency of the TCMEP in monitoring the facial nerve. To this aim, they performed TES technique on 52 patients under the CPA surgery. Similar to our study, the facial status was predicted immediately after the surgery using the HP scale and the changes of electromyographic responses of stimulated muscle before and after the tumor removal. The obtained results of the mentioned study showed that the changes of the electromyographic responses of the facial nerve immediately after the surgery should not be reduced to more than $57 \%$, compared to that of the pre-surgery. Therefore, they concluded that more than $57 \%$ changes in the facial nerve may cause problems in the long-term. ${ }^{7}$ The weakness of the TCMEP approach is its intermittent feature since this approach is performed every $10 \mathrm{~min}$; therefore, the use of the EMG is constantly suggested. The EMG can present a feedback from the environmental damages, which have shown to have a significant correlation with surgery outcomes in terms of the facial nerve. ${ }^{14}$ The FNMEP is a standard approach confirmed by the National Institutes of Health, USA, as a proper technique to avoid damaging the facial nerve and facilitate long-term prediction of its performance. In our study, this method was used to predict the facial nerve status after the operation. In a study conducted by Fukuda et al., the FNMEP was used, and the corkscrew was installed on the $\mathrm{C} 3, \mathrm{C} 4$, and $\mathrm{Cz}$ regions. The motor responses of the orbicularis oris and oculi were recorded, which were caused by180-550 V supramaximal stimulations. In the mentioned study, there was a correlation between the post-surgery facial nerve function and FNMEP ratios. Accordingly, in case the FNMEP ratio remained > 50\%, the patient had a satisfactory facial nerve function. ${ }^{13}$ Our findings demonstrated that the intraoperative FNMEP monitoring could be useful for predicting the facial nerve function post-surgery. This new method is a valuable adjunct to the conventional facial nerve monitoring techniques. Totally, intraoperative monitoring is an indispensible part of the acoustic neuroma surgery that leads to significant improvements in the functional outcomes of CPA surgery and prevents the facial paralysis. ${ }^{15}$ According to our results, severe facial nerve dysfunction was observed immediately after the surgery in about one third of the patients. However, the facial function was fully recovered in about one third of cases in the followup period; accordingly, these patients obtained HB grade I or II at the follow-up evaluation. Similar to our study, in the study carrieod out by Cosetti et al, complete paralysis and severe facial nerve dysfunction were observed in $32 \%$ of the patients immediately after the operation. Furthemore, the facial function was recovered in more than one fifth of the patients. ${ }^{7}$ Temporary motor nerve conduction block, neuropraxia or axonotmesis, edema, vasoconstriction, and herpes virus reactivation are introduced as the main causes of immediate paresis or paralysis in the patients with CPA tumor; however, the etiology of this problem have not fully discovered yet. Some of these causes (e.g., conduction block) may rapidly improve; nevertheless, in some cases, the paresis or paralysis may persist for months and even, as can be seen in case of neuropraxia or axonometsis, the patient may not achieve the pre-operative function of facial nerve. ${ }^{16}$ Immediately after surgery, the occurance of facial nerve paralysis or enhancement of HB score is possible; however, the causes are not clear. These conditions may be due to neurapraxia and temporary conduction block of the facial nerve or perioperative trauma, which are usually recovered a day or two after the surgery. Nonetheless, neurapraxia and axonotmesis injuries may continue for a few months. ${ }^{9}$ Since the CPA surgery may be associated with main communication problems and ocular complications, the recognition of the patients at risk is very important due to the necessity of the prevention of such complications in these patients. The relationship between TCMEP, HB grading, and other variables, such as age, tumor size, diagnosis time, and preoperative symptoms, are not clear yet. In our study, the investigation of the association between the TCMEP and other approaches showed a positive correlation between the HB score and amplitude responses of FNAP and TES. The predictive value of these variables in terms of the facial nerve are confirmed by similar studies ${ }^{16,17}$ The outcome of the patients with CPA is largely affected by the rate of tumor adhesion to the facial nerve sheath and facial nerve, which was not possible to check in this study. However, the relationship between these variables was assessed in our study. The data on facial nerve function cannot be achieved through continuous EMG or direct electrical stimulation. Nonetheless, the presence of a stimulating probe is necessary to gain information during the surgery, which can be transferred by the TCMEP monitoring. In the present study, the BAEP approach resulted in the improvement of the hearing quality and speech discrimination six months after the operation. This finding was also confirmed in a study conducted by Yamakami et al. ${ }^{18}$ However, in a study carried out by Rampp et al., the auditory steady-state response was suggested as a more effective tool in the assessment of the hearing quality and speech discrimination. ${ }^{19}$. One of the advantages of BAEP approach is the low probability of the anesthetics effectiveness on the BAEP responses. As the findings of this study revealed, the TCMEP was a useful method for monitoring the facial nerve during the surgery, which could predict the facial nerve complications along with continuous EMG. Therefore, this method cannot be considered as a complete technique for monitoring the facial nerve during the CPA surgery since it should be simultaneously used along with other monitoring approaches. However, the TCMEP 
approach is more favorable for monitoring the facial nerve in the posterior fossa surgery.

\section{List of acronyms}

CPA: Cerebellopontine Angle

HB: House-Brackmann

EMG: Electromyography

FNAPs: Facial Nerve Antidromic Potentials

TES: Transcranial Electrical Stimulation

BAEPs: Brainstem Auditory Evoked Potentials

TCMEP: Transcranial motor evoked potential

ATH: Absolute threshold of hearing

SDS: Speech discrimination score

IOM: Intraoperative neurophysiological monitoring

MEP: The motor evoked potentials

\section{Author's contributions}

Each author contributed in equal part to the manuscript.

\section{Acknowledgments and Funding}

Hereby, we extend our gratitude to the Deputy of Research and Technology as well as all the members and experts of the Research Council of Mashhad University of Medical Sciences for approving the project proposal. We also wish to appreciate all the patients participating in this study. Other thanks go to the personnel of operating room and anesthesiology department of the hospital. Additionally, we would like to thank Dr. A.R. Ashtiani, from department of Neurology for his cooperation. Funding: None.

\section{Conflict of Interest}

The authors declare no conflicts of interests derived from the outcomes of this stud.

\section{Ethical Publication Statement}

We confirm that we have read the Journal's position on issues involved in ethical publication and affirm that this report is consistent with those guidelines.

\section{Corresponding Author}

Ali Nazemi Rafie, Department of Neurosurgery, Arak University of Medical Sciences, Arak, Iran.

Email: nazemirafie.ali@gmail.com

\section{E-mails of co-authors}

Mohsen Dalvandi: Mdalvandi@yahoo.com Alireza Kamali: Alikamaliir@yahoo.com Alireza Jamshidifard: arjfard@yahoo.com

\section{References}

1. Son DW, Choi CH, Cha SH. Epidermoid tumors in the cerebellopontine angle presenting with trigeminal neuralgia. J Korean Neurosurg Soc 2010;47:271-7. doi: 10.3340/jkns.2010.47.4.271. Epub 2010 Apr 30.

2. Nakamura M. Facial and cochlear nerve function after surgery of cerebellopontine angle meningiomas. Samii's Essentials in Neurosurgery, Springer 2014:321-32.

3. House JW, Brackmann DE. Facial nerve grading system. Otolaryngol Head Neck Surg.1985;93(2):146-7.

4. Acioly MA, Liebsch $\mathrm{M}$, Carvalho $\mathrm{CH}$, et al. Transcranial electrocortical stimulation to monitor the facial nerve motor function during cerebellopontine angle surgery. Neurosurgery 2010;66:354-62.

5. Fehlings MG, Brodke DS, Norvell DC, Dettori JR. The evidence for intraoperative neurophysiological monitoring in spine surgery: does it make a difference? Spine 2010;35:S37-S46.

6. Lall RR, Lall RR, Hauptman JS, et al. Intraoperative neurophysiological monitoring in spine surgery: indications, efficacy, and role of the preoperative checklist. Neurosurgical focus 2012;33:E10.

7. Cosetti MK, Xu M, Rivera A, et al. Intraoperative transcranial motor-evoked potential monitoring of the facial nerve during cerebellopontine angle tumor resection. J Neurol Surg B Skull Base. 2012;73:308-15. doi: 10.1055/s-0032-1321507.

8. Galloway GM, Nuwer MR, Lopez JR, Zamel KM. Intraoperative neurophysiologic monitoring: Cambridge University Press, 2010.

9. Kim S-M, Kim SH, Seo DW, Lee KW. Intraoperative neurophysiologic monitoring: basic principles and recent update. J Korean Med Sci 2013;28:1261-9. doi: 10.3346/jkms.2013. 28.9.1261. Epub 2013 Aug 28.

10 Mosleh M. Development and evaluation of a speech recognition test for Persian speaking adults. Bimonthly Audiology - Tehran University of Medical Sciences. 2001;9:72-6.

11. Akagami R, Dong CC, Westerberg BD. Localized transcranial electrical motor evoked potentials for monitoring cranial nerves in cranial base surgery. Neurosurgery. 2005;57:78-85. discussion 78-85.

12. Dong CC, MacDonald DB, Akagami R, et al. Intraoperative facial motor evoked potential monitoring with transcranial electrical stimulation during skull base surgery. Clin neurophysiol 2005;116:588-96.

13. Fukuda M, Oishi M, Takao T, et al. Facial nerve motor-evoked potential monitoring during skull base surgery predicts facial nerve outcome. J Neurol Neurosurg Psychiatry 2008;79:1066-70. doi: 10.1136/jnnp.2007. 130500. Epub 2008 Feb 1.

14. Prell J, Rachinger J, Scheller C, et al. A Real-Time Monitoring System for the Facial Nerve. Neurosurgery 2010;66:1064-73.

15. Youssef AS, Downes AE. Intraoperative neurophysiological monitoring in vestibular schwannoma surgery: advances and clinical implications. Neurosurgical focus 2009;27(4):E9. 


\section{Prognostic Value of Multimodal Intraoperative Monitoring}

Eur J Transl Myol 28 (1): 124-131, 2018

16 Bernat I, Grayeli AB, Esquia G, et al. Intraoperative electromyography and surgical observations as predictive factors of facial nerve outcome in vestibular schwannoma surgery. Otol Neurotol 2010;31:306-12. doi: 10.1097/MAO. 0b013e3181be6228.

17. Esquia-Medina GN, Grayeli AB, Ferrary E, et al. Do facial nerve displacement pattern and tumor adhesion influence the facial nerve outcome in vestibular schwannoma surgery? Audiol Neurootol 2013;18:406-13. doi: 10.1159/000 354115. Epub 2013 Oct 23.

18. Yamakami I, Ushikubo O, Uchino $\mathrm{Y}$, et al. Intraoperative monitoring of hearing function in the removal of cerebellopontine angle tumor: auditory brainstem response and cochlear nerve compound action potential. No shinkei geka Neurological surgery 2002;30:275-82.

19. Rampp S, Rensch L, Simmermacher S, et al. Intraoperative auditory steady-state monitoring during surgery in the cerebellopontine angle for estimation of postoperative hearing classes. J Neurosurg 2017;127:559-568. doi: 10.3171/2016.7.JNS16460. Epub 2016 Oct 14.

Received for publication: January 25, 2018

Revision received: February 5, 2018

Accepted for publication: February 7, 2018 